\title{
Genome sequencing, annotation and analysis of Salmonella enterica sub species salamae strain DMA-1
}

Sathyaseelan Sathyabama ${ }^{2 \dagger}$, Gurwinder Kaur ${ }^{1+}$, Amit Arora', Sheenam Verma ${ }^{2}$, Nida Mubin $^{2}$, Shanmugam Mayilraj ${ }^{1^{*}}$ and Javed N Agrewala $2^{2^{*}}$

\begin{abstract}
Background: The genus Salmonella is Gram-negative which belongs to the family Enterobacteriaceae. In this study, we have sequenced the whole genome of the strain DMA-1, which was isolated from mouse stool sample and identified as Salmonella enterica subspecies salamae.

Results: The strain DMA-1 was closely related at the 16S rRNA gene sequence level with the members of the genus Salmonella: Salmonella enterica subspecies salamae DSM $9220^{\top}(100 \%)$, followed by Salmonella enterica subspecies diarizonae (99.1\%), Salmonella enterica subspecies enterica (99.0\%) and Salmonella enterica subspecies indica (98.5\%). We obtained the draft genome of $S$. enterica subspecies salamae strain DMA-1 with a size of 4,826,209 bp and mean $\mathrm{G}+\mathrm{C}$ content of $52.0 \mathrm{~mol} \%$.

Conclusions: We for the first time, sequenced the entire genome of the strain DMA-1 which was isolated from the mouse stool sample and identified it as Salmonella enterica, sub species salamae. Further, we subjected the whole genome sequencing data for annotation that revealed several genes responsible for the pathogenesis, virulence, defense, metabolism and other genomic features.
\end{abstract}

Keywords: Salmonella enterica subspecies salamae, RAST, EzTaxon

\section{Background}

During a study on identifying bacterial diversity of mouse stool samples, strain DMA-1 was isolated on tryptone soya agar (TSA, HiMedia, Mumbai, India). The strain DMA-1 was subjected to polyphasic taxonomic studies to identify the exact taxonomic status. The polyphasic taxonomical studies involved phenotypic, biochemical characterization and 16S rRNA gene sequencing that identified it as Salmonella enterica subspecies salamae. The genus Salmonella was first proposed in 1952 by Kauffmann and Edwards [1]. Later on, it was emended in 1987 by Le Minor and Popoff [2]. The members of the genus Salmonella are Gram-negative, rod shaped, facultative anaerobes capable of aerobic respiration producing

\footnotetext{
*Correspondence: mayil@imtech.res.in; javed@imtech.res.in

${ }^{\dagger}$ Equal contributors

'Microbial Type Culture Collection and Gene bank (MTCC), CSIR-Institute of Microbial Technology, Sector 39-A, Chandigarh, India

${ }^{2}$ Immunology Laboratory, CSIR-Institute of Microbial Technology, Sector 39-A, Chandigarh, India
}

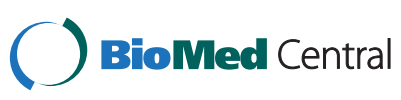

(c) 2014 Sathyabama et al.; licensee BioMed Central Ltd. This is an Open Access article distributed under the terms of the Creative Commons Attribution License (http://creativecommons.org/licenses/by/4.0), which permits unrestricted use, distribution, and reproduction in any medium, provided the original work is properly credited. The Creative Commons Public Domain Dedication waiver (http://creativecommons.org/publicdomain/zero/1.0/) applies to the data made available in this article, unless otherwise stated.
ATP and fermentation in the absence of oxygen. At present, the genus Salmonella consists of three species and six sub species. The Salmonella enterica subspecies include: S. enterica subspecies enterica (subspecies I), S. enterica subspecies salamae (subspecies II), S. enterica subspecies arizonae (subspecies IIIa), S. enterica subspecies diarizonae (subspecies IIIb), S. enterica subspecies houtenae (subspecies IV) and S. enterica subspecies indica (subspecies V). S. enterica includes the majority of Salmonella strains isolated from humans and warm blooded animals. But Salmonella bongori is typically obtained from cold blooded animals [3]. Most of the rodents present in laboratories are susceptible to Salmonella infections. Rodent feces cause infections via fecal-oral transmission, when a susceptible rodent ingests the bacteria present in their feed or contaminated drinking water. Immunocompromised caretakers of these rodents may get serious illness, which in some cases develop into life threatening diseases. Therefore in laboratories incoming rodents are 
quarantined and health monitoring for any rodent colony involves routine intestinal culture [4]. Infection caused by Salmonella enterica species is a major public health issue inflicting mortality globally. Contagious diseases caused by $S$. enterica depend mainly on their secreted proteins and adhesion from fimbrial and non-fimbrial sources, which produce biofilm and build contact with the host cell [5]. S. enterica also contains type I fimbriae, representative of other enteric bacteria, which help in attaching themselves to epithelial cells [6]. They are shorter in size than flagella but have a peritrichous distribution. Fimbriae are made up of major and minor protein subunits that make a cylinder around a hollow core [7]. Not all strains of S. enterica express fimbriae, but the fimbriaeted strains are more virulent [8].

Salmonella species could be differentiated on the basis of three antigens present on the cell: $\mathrm{H}$ antigen which is a flagellar antigen occurring only in one phase ( 1 or 2$)$ and can interchange itself $[9,10]$; $\mathrm{O}$ antigen is a somatic antigen present on the outer membrane. The specificity of the $\mathrm{O}$ antigen is obtained by the character of the repeating units present on the outer O-polysaccharide chain [9]. The Vi antigen, virulence antigen which is a capsular polysaccharide that overlays the $\mathrm{O}$ antigen, but it is not possessed by all serovars. The capsule present in the Salmonella sp. is not essential for infection, yet it increases the infectivity by making it less visible to the body's immune system [11].

S. enterica is also able to extract iron from host proteins by siderophores, which are formed by the cell when iron concentration is low, siderophores also contribute to virulence [12]. It also shows a mixed-acid heterofermentation of glucose to produce ATP. This process also produces $\mathrm{CO}_{2}$ and $\mathrm{H}_{2}$ with a variety of acid final -products, for example formate, acetate, lactate, and succinate [13]. S. enterica can nourish on maltose and maltodextrins with the help of type I ATP-binding cassette transporter. This system is present on a maltose regulon. In a maltose regulon the maltose enter into the cell by crossing the outer membrane through a homotrimeric maltoporin, after that it binds to a protein having pore-forming subunits that result in the translocation of an ATP-binding subunit across the inner-membrane where it is used by the cell [14]. S. enterica causes infectious diseases by entering the host cells that are present around the intestine, and attaching themselves to the cells of intestine, thereby causing ruffling response in the host cell membrane [5]. Ruffling is linked to a triggering response that results in macropinocytosis. The vesicles of macropinocytosis are large, and provide an efficient route for non-selective endocytosis of macromolecules [15]. The entry of the bacterial cells also harms the microvilli which are present on the cell surface, which causes disturbance in the white blood cells as they start to flood the mucosa thereby altering the homeostasis between absorption and secretion of the body [16].

S. enterica has a number of virulence factors like enterotoxin, endotoxin, and cytotoxin which are responsible for virulence. Enterotoxin causes diarrhea and vomiting in which the cells discharge huge amounts of fluid into the lumen. Endotoxin consists of toxic lipopolysaccharide which causes fever by inspiring host cells to discharge endogenous pyrogens. The cytotoxin interferes with protein synthesis in the host cell and leads to an efflux of calcium ions [15]. In some cases the infectious cells are transferred to the liver or spleen, where they multiply and return either to the host's intestinal tract or get defecated [9].

Food contamination often occurs from infested feces that are present in polluted water, soil or other contaminated environments. S. enterica is responsible for causing many abdominal complications like gastroenteric disorders which involve stomach pain, cramps etc. When there is an imbalance between absorption and secretion, first signs include abdominal cramps, diarrhea, fever and nausea. Mostly the infection caused by food contamination cures within one week's time, but more serious cases generally need fluoroquinolone or cephalosporin's that eliminate $S$. enterica by interfering with their cell wall synthesis [17].

Presently only two species of the genus Salmonella have been sequenced for their whole genome: Salmonella bongori (two isolates), Salmonella enterica, forty three isolates including two isolates of Salmonella enterica subsp salamae, isolated from different sources, Salmonella enterica subspecies salamae serovar 58:1, z13, z38:z6 strain 000163 [18] and Salmonella enterica subspecies salamae strain 3588/07 [Young et al. unpublished]. It is for the first time whole genome of strain DMA-1, isolated from mouse stool sample was sequenced by us. The whole genome sequencing was done in order to produce accurate reference genome which aids in identification and comparative genomic study. Moreover it also helps to analyze genes involved in virulence, disease and multidrug resistance.

\section{Methods}

Isolation, identification, DNA extraction, genome sequencing, assembly and annotation

Salmonella enterica subspecies salamae strain DMA-1 was isolated from mouse stool samples on $9^{\text {th }}$ October, 2012, and grown in tryptone soya agar (TSA, HiMedia, Mumbai, India). Genomic DNA extraction, amplification, 16S rRNA gene sequencing, phylogenetic analysis and $\mathrm{G}+\mathrm{C}$ content were performed as described previously $[19,20]$. The genome of strain Salmonella enterica, subspecies salamae strain DMA-1 was sequenced at cCAMP, Next Generation Genomic Facility, Bengaluru, India (http://www.ccamp.res.in), using a standard run of Illumina HiSeq 1000 sequencing technology. CLC Bio Workbench v6.0.2 (CLC Bio, Aarhus, Denmark) was employed for preprocessing of the data to remove the redundant and irrelevant information. A high quality $24,334,734$ vector filtered reads at approximately 497.37 
times coverage were used for final assembly (at word size of 45 and bubble size of 98). This draft genome comprising 4,826,209 bp was annotated with the help of RAST (Rapid Annotation using Subsystem Technology) system [21] server and RNAmmer 1.2 [22] server.

\section{Results and discussions}

Quality assurance: Identification

Based on 16S rRNA gene sequence and phylogenetic analysis, strain DMA-1 was identified as Salmonella enterica subspecies salamae. Further polyphasic taxonomical data confirmed that the strain DMA-1 belongs to Salmonella enterica subspecies salamae. After two days of incubation at $30^{\circ} \mathrm{C}$ the colonies of the strain DMA-1 on TSA were cream colored and about 1-3 mm in diameter, circular, smooth, glistening, opaque, and convex with an entire margin. Cells were short-rod shaped, catalase-positive and oxidase-negative. Growth temperature ranged from $20^{\circ} \mathrm{C}$ and $37^{\circ} \mathrm{C}$, optimum growth temperature was $30^{\circ} \mathrm{C}$, no growth occurred at $42^{\circ} \mathrm{C}$. $\mathrm{pH}$ range for growth was $5.2-7.5$. $\mathrm{NaCl}$ was tolerated up to $2 \%$. It was positive for hydrolysis of gelatin, production of hydrogen sulphide. It was negative for casein, starch hydrolysis, indole, urease, methyl red, VogesProskauer test and nitrate reduction. Acid was produced from D-glucose, D-dulcitol, and sorbitol but not from salicin and lactose. The $\mathrm{G}+\mathrm{C}$ content of the genomic DNA was $52.0 \mathrm{~mol} \%$. A neighbour-joining tree (Figure 1) based on 16S rRNA gene sequence of the strain DMA-1 shows the phylogenetic relationship among the species of genus Salmonella and the organism formed a distinct branch along with Salmonella enterica subspecies salamae separated from the other members of the genus Salmonella.

\section{Initial Findings: Genomic features}

The draft genome of Salmonella enterica subspecies salamae strain DMA-1 composed of 4,826,209 bp with
G+C content of 52.0 mol\%, 4,322 predicted CDSs and 69 RNAs. The final assembly contains 32 contigs with $\mathrm{N}_{50}$ contig length of $4,24,412 \mathrm{bp}$; the largest contig assembled measured 6,24,758 bp. RAST annotation indicates that Salmonella enterica subspecies enterica serovar typhimurium strain SL 1344 (score 519), Salmonella enterica subspecies enterica serovar typhi Ty2 (score 449), Salmonella enterica subspecies enterica serovar strain CVM 29188 (score 424) and Salmonella enterica (score 416) are the closest neighbors of the strain DMA-1. Summary of the basic characteristic features of the genomes is given in Table 1 . The sub-system distribution of the strain DMA-1 is represented in Figure 2. Circular genome map of strain DMA-1 Salmonella enterica subspecies salamae showing the major genes and their regulators is shown as Figure 3.

\section{Genes involved in virulence, disease and defense, resistance to antibiotics and toxic compounds and multidrug resistance efflux pumps}

Whole genome annotation at RAST server shows that Salmonella enterica, subspecies salamae strain DMA1 contains genes related to the virulence, disease, defense and adhesion; mediator of hyperadherence YidE in Enterobacteria and its conserved region; $16 \mathrm{kDa}$ heat shock protein A and B, outer membrane lipoprotein YidQ and HTH-type transcriptional regulator YidP. Bacteriocins, ribosomally synthesized antibacterial peptides; colicin $\mathrm{V}$ and bacteriocin production cluster; acetyl-coenzyme A carboxyl transferase beta chain (EC 6.4.1.2), amidophosphoribosyltransferase (EC 2.4.2.14), colicin V production protein, DedA protein, DedD protein, dihydrofolate synthase (EC 6.3.2.12), folylpolyglutamate synthase (EC 6.3.2.17) and tRNA pseudouridine synthase A (EC 4.2.1.70). Resistance to antibiotics and toxic compounds; the mdtABCD multidrug resistance cluster, multidrug transporter $\mathrm{MdtC}$, response regulator BaeR,

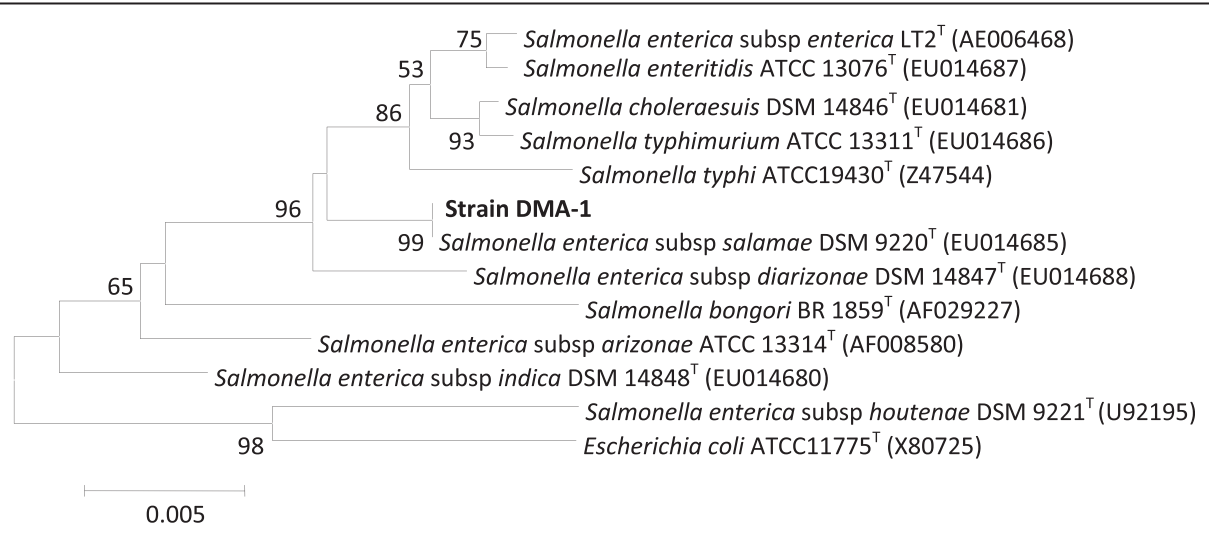

Figure 1 Phylogenetic neighbour-joining tree based on 16S rRNA gene sequences showing the relationship between strain DMA-1 and related members of the genus Salmonella. Escherichia coli (X80725) was used as an out-group. Bootstrap values (expressed as percentages of 100 replications) greater than 50\% are given at nodes. Bar 0.005\% sequence variation. GenBank accession numbers are given in parentheses. 
Table 1 Characteristic features of the annotated strain DMA-1 Salmonella enterica, sub species salamae

\begin{tabular}{llll}
\hline Nucleotide & Count & Frequency (\%) & $\mathbf{G + C ~ m o l \%}$ \\
\hline Adenine (A) & $1,155,424$ & 23.9 & \\
Cytosine (C) & $1,265,442$ & 26.2 & 52.0 \\
Guanine (G) & $1,246,212$ & 25.8 & \\
Thymine (T) & $1,157,744$ & 24.0 & \\
Any nucleotide (N) & 1,387 & 0.0 & \\
\hline Characters & & Length (bp) & \\
\hline $\mathrm{N}_{75}$ & & $2,80,250$ & \\
$\mathrm{~N}_{50}$ & $4,24,412$ & \\
$\mathrm{~N}_{25}$ & $5,39,878$ & \\
Minimum & & 1,046 & \\
Maximum & & $6,24,758$ & \\
Average & & $1,50,819$ & \\
Count & 32 & \\
Total & & $4,826,209$ \\
\hline
\end{tabular}

multidrug transporter MdtB, probable RND efflux membrane fusion protein, multidrug transporter MdtD and sensory histidine kinase BaeS. Multiple antibiotic resistance MAR loci; multiple antibiotic resistance protein MaeR, MaeA, MaeB and MaeC. Multidrug resistance efflux pumps; outer membrane lipoprotein NodT family, membrane fusion protein CmeA, outer membrane lipoprotein $\mathrm{CmeC}$, multidrug and toxin extrusion (MATE) family efflux pump YdhE/NorM, macrolide export ATPbinding/permease protein $\mathrm{MacB}, \mathrm{MacA}$ protein and type I secretion outer membrane protein, TolC precursor.

\section{Phages and prophages}

The following proteins were identified in the genome of strain DMA-1: phage outer membrane lipoprotein Rz1, phage holin, class II, spanin Rz, lytic protein $\mathrm{Rz}$, coactivator of prophage gene expression IbrA and IbrB, tail assembly protein, Nin protein, anti-termination protein Q, major capsid protein, capsid scaffolding protein and NinB DNA recombination.

\section{Functional based differential comparative genomic analysis}

Functional based comparative genomic analysis for strains Salmonella enterica subspecies salamae DMA-1 and Salmonella enterica subspecies enterica SL483 were analyzed with the help of RAST (Rapid Annotation using Subsystem Technology) system. We identified a total of 3,437 genes (Additional file 1: Table S1) in which only 45 genes (Additional file 2: Table S2) were different between these two strains, based on their category, subcategory, subsystem and role. Some of the major differences include, the genes for category: virulence disease and defense having subcategory: adhesion, play role as $16 \mathrm{kDa}$ heat shock protein $\mathrm{B}$, mediator of hyperadherence YidE, outer membrane lipoprotein YidE, uncharacterized proteinYidR which are present in DMA-1 but absent in S. enterica subspecies enterica. Similar to this there are genes for category: phages, prophages, transposable elements, plasmids and having subcategory: phages, prophages having role as phage terminase small subunit, phage replication protein which are absent in S. enterica subspecies enterica.

\section{Conclusion}

It was for the first time, whole genome sequencing of the strain DMA-1, isolated from mouse stool sample was carried out by us and the strain was identified as Salmonella enterica sub species salamae. Further, the genome sequencing data was subjected to annotation and the analysis revealed the genes responsible for the pathogenesis, virulence, defence, metabolism and other genomic features. The whole genome of $S$. enterica sub species salamae comprised of 4,826,209 bp in size with a $\mathrm{G}+\mathrm{C}$ content of $52.0 \mathrm{~mol} \%$, having 4,322 predicted CDSs and 69 RNAs. The final assembly contained 32 contigs of

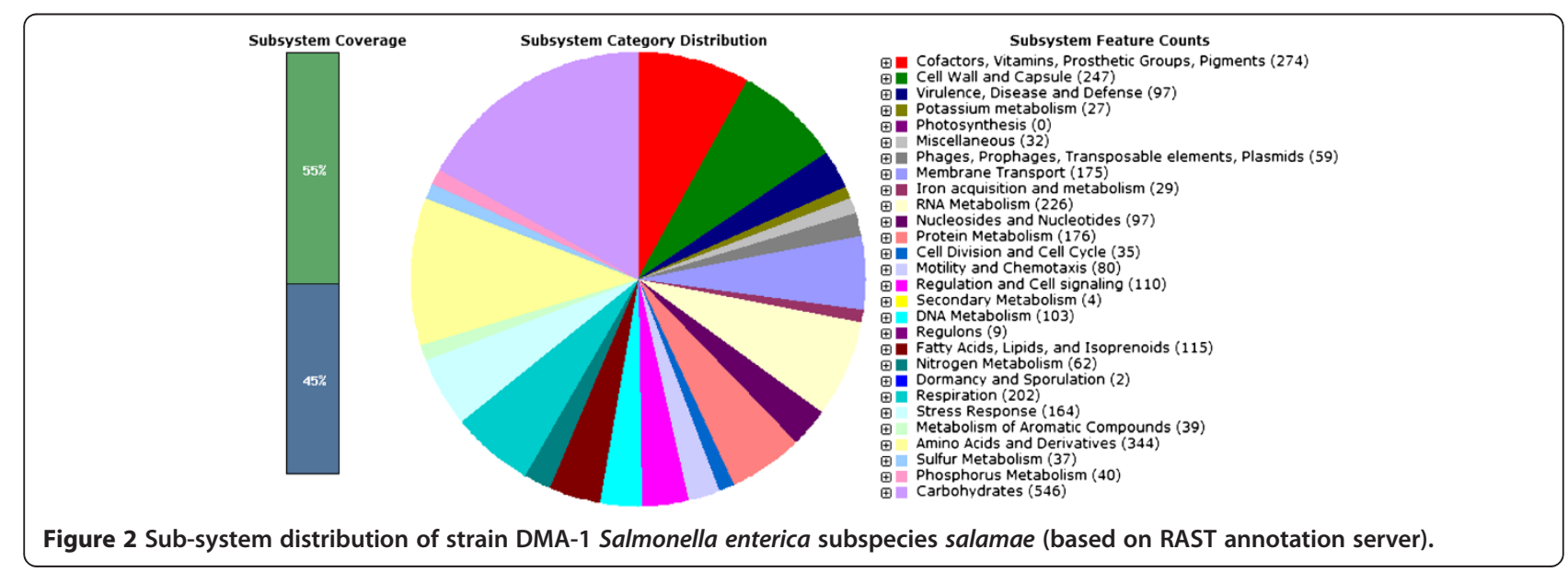




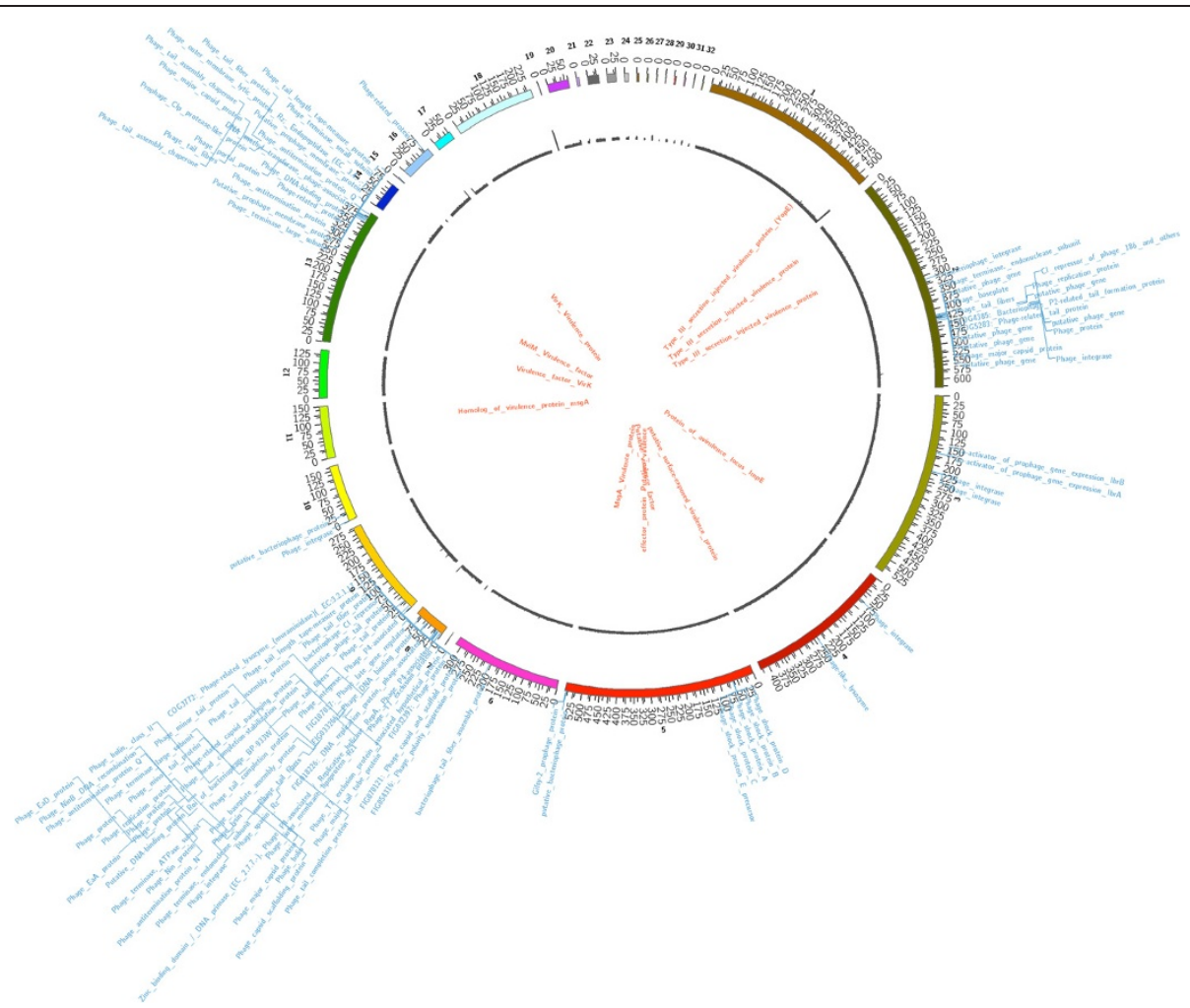

Figure 3 Circular genome map of strain DMA-1 Salmonella enterica subspecies salamae showing the major genes and their regulators. The 32 assembled contigs are shown by different coloured ideograms having their base-pair positions depicted at a scale of 1000 units. The coverage of the assembly at each base pair can be seen by the grey coloured track. Annotations descriptors for "virulent" genes [inner label: red] and "phage" related genes [outer label: blue] are mapped onto their respective contig positions. As many of the annotation descriptors occupy neighbouring positions on the contigs, the descriptors are stacked to allow better visualization.

total size $4,826,209$ bp with $\mathrm{N}_{50}$ contig length of $4,24,412 \mathrm{bp}$; the largest contig assembled measured $6,24,758 \mathrm{bp}$.

\section{Future directions}

There have been regular reports of Salmonella enterica subspecies salamae associated infections in Indian subcontinent. Therefore it becomes imperative to study the pathogenicity of the organism and its variants, which are very less explored and understood. The future work focuses on collecting various isolates of Salmonella enterica subsp salamae pertaining to different geographical locations across India, and to study their genome extensively. Whole genome sequencing of Salmonella enterica subspecies salamae would help in a deeper understanding of its pathogenicity, which enables us to identify new and improved drug targets for Salmonella associated diseases.

\section{Ethical clearance}

The study was ethically approved by the Institutional Biosafety Committee (Ref/IBSC/2012-2/09) and Institutional Animal Ethics Committee (IAEC 13/01) of the CSIRInstitute of Microbial Technology, Chandigarh, India.

\section{Availability of supporting data}

This Whole Genome Shotgun project has been deposited at DDBJ/EMBL/GenBank under the accession ATFA00000000. The version described in this paper is the first version, ATFA01000000.

\section{Additional files}

Additional file 1: Table S1. Functional based differential comparative genomic analysis of (1) Salmonella enterica subspecies salamae strain DMA-1 and (2) Salmonella enterica subspecies enterica strain SL 483.

Additional file 2: Table S2. Functional based (Virulence, Disease and Defense and Phages, Prophages, Transposable elements, Plasmids) differential comparative genomic analysis of (1) Salmonella enterica subspecies salamae strain DMA-1 and (2) Salmonella enterica subspecies enterica strain SL 483.

\section{Competing interests}

The authors have no competing interests.

\section{Authors' contributions}

Performed experiments: GK, SS, AA, SV and NM. Planned and executed experiments analyzed data and wrote manuscript: JNA and SM. All authors read and approved the final manuscript. 


\section{Acknowledgments}

This work was supported by CSIR net project Human Microbiome (HUM), CSIR-Institute of Microbial Technology, Chandigarh, India. This is IMTECH communication number 08/2014.

Received: 7 March 2014 Accepted: 3 April 2014 Published: 11 April 2014

\section{References}

1. Kauffmann F, Edwards PR: Classification and nomenclature of Enterobacteriaceae. Int Bull Bacteriol Nomend Taxon 1952, 2:2-8.

2. Le Minor L, Popoff MY: Request for an Opinion. Designation of Salmonella enterica sp. nov., nom. rev., as the type and only species of the genus Salmonella. Int J Syst Bacteriol 1987, 37:465-468.

3. Nataro JP, Bopp CA, Fields PI, Kaper JB, Strockbine NA: Escherichia, Shigella and Salmonella. In Manuals of clinical microbiology, Volume 1. 10th edition. Edited by Versalovic J. Washington DC: ASM Press; 2011.

4. Healing TD: Salmonella in rodents: a risk to man? CDR (Lond Engl Rev) 1991, 1(10):R114-R116.

5. Hensel M: "Secreted Proteins and Virulence in Salmonella enterica". Bacterial Secreted Proteins: Secretory Mechanisms and Role in Pathogenesis. Caister Academic Press; 2009. ISBN 978-1-904455-42-4.

6. Madigan M, Martinko J, Dunlap P, Clark D: Brock Biology of Microorganisms. Pearson Education: San Francisco (CA); 2009

7. Thorns C, Woodward M: Fimbriae of Salmonella. In Salmonella in Domestic Animals. Edited by Wray C, Wray A. New York: CABI Publishing; 2000:35-55.

8. Mahon CR, Manuselis G. Enterobacteriaceae. In Textbook of Diagnostic Microbiology. Edited by Mahon CR, Manuselis G. Philadelphia: W.B. Saunders; 1995.

9. Giannella RA: Salmonella. In Medical Microbiology. 4th edition. Edited by Barron S. Galvaston (TX): University of Texas Medical Branch; 1996.

10. Yamamoto S, Kutsukake K: FljA-Mediated Post transcriptional Control of Phase 1 Flagellin Expression in Flagellar Phase Variation of Salmonella enterica Serovar typhimurium. J Bacteriol 2006, 188(3):958-967.

11. Wain J, House D, Zafar A, Baker S, Nair S, Kidgell C, Bhutta Z, Dougan G, Hasan R: Vi Antigen Expression in Salmonella enterica Serovar Typhi Clinical Isolates from Pakistan. J Clin Microbiol 2005, 43(3):1158-1165.

12. Peterson J: Bacterial Pathogenesis. In Medical Microbiology. 4th edition. Edited by Barron S. Galvaston (TX): University of Texas Medical Branch; 1996.

13. Jurtshuk P: Bacterial Metabolism. In Medical Microbiology. 4th edition. Edited by Baron S. Galveston (TX): University of Texas Medical Branch; 1996.

14. Bordignon E, Grote M, Schnelder E: The maltose ATP-binding cassette Transporter in the 21st century- towards a structure dynamic perspective on its mode of action. Mol Microbiol 2010, 77(6):1354-1366

15. Swanson JA, Watts C: Macropinocytosis. Trends Cell Biol 1995, 5.11:424-428. Web. 16 Mar. 2011

16. McCormick B, Miller S, Carnes D, Madara J: Transepithelial Signaling to Neutrophils by Salmonellae: a novel Virulence Mechanism for Gastroenteritis. Infect Immun 1995, 63(6):2302-2309.

17. Centers for Disease Control and Prevention: Salmonella.; 2011. Web. 16 Mar 2011. Division of Foodborne, Waterborne and Environmental Diseases.

18. Timme RE, Allard M, Luo Y, Strain E, Pettengill J, Li C, Ottesen A, Brown E: Phylogenetic Diversity of the Enteric Pathogen Salmonella enterica sunsp. enterica Inferred from Genome-Wide Reference -Free SNP Characters. Genome Biol Evol 2013, 5(11):2109-2123.

19. Mayilraj S, Saha P, Suresh K, Saini HS: Ornithinimicrobium kibberense sp. nov., isolated from the Himalayas, India. Int J Syst Evol Microbiol 2006 56:1657-1661.

20. Singh NK, Raichand R, Kaur I, Kaur C, Pareekh S, Mayilraj S: Description of a novel bacterium Exiguobacterium himgiriensis sp. nov., isolated from soil sample collected from the cold desert of The Indian Himalayas. Antonie Van Leeuwenhoek 2013, 103:789-796.
21. Aziz RK, Bartels D, Best AA, DeJongh M, Disz T, Edwards RA, Formsma K, Gerdes S, Glass EM, Kubal M, Meyer F, Olsen GJ, Olson R, Osterman AL, Overbeek RA, McNeil LK, Paarmann D, Paczian T, Parrello B, Pusch GD, Reich C, Stevens R, Vassieva O, Vonstein V, Wilke A, Zagnitko O: The RAST Server: rapid annotations using subsystems technology. BMC Genomics 2008, 9:75

22. Lagesen $\mathrm{K}$, Hallin P, Rodland EA, Staerfeldt HH, Rognes T, Ussery DW: RNAmmer: consistent and rapid annotation of ribosomal RNA genes. Nucleic Acids Res 2007, 35:3100-3108.

doi:10.1186/1757-4749-6-8

Cite this article as: Sathyabama et al:: Genome sequencing, annotation and analysis of Salmonella enterica sub species salamae strain DMA-1. Gut Pathogens 2014 6:8.

\section{Submit your next manuscript to BioMed Central and take full advantage of:}

- Convenient online submission

- Thorough peer review

- No space constraints or color figure charges

- Immediate publication on acceptance

- Inclusion in PubMed, CAS, Scopus and Google Scholar

- Research which is freely available for redistribution

Submit your manuscript at www.biomedcentral.com/submit
C Biomed Central 